\title{
Le problème de la coordination dans la controverse Hayek-Keynes
}

\author{
Abdallah Zouache ${ }^{*}$ \\ CREUSET, Université de Saint-Étienne**
}

\begin{abstract}
«On se souvient à peine du temps où les nouvelles théories de Hayek étaient les principales rivales de celles de Keynes. A qui fallait-il donner raison, à Keynes ou Hayek? "
\end{abstract}

Hicks, 1967, p.203

\section{Introduction : le contexte de la controverse}

La controverse Hayek-Keynes se déroule dans le contexte des « années de haute théorie » (Shackle (1967)) qui témoigne de la vivacité des échanges intellectuels. C'est pour combattre les idées de Keynes - exposées dans le Traité sur la Monnaie édité en décembre 1930 - que Robbins invite Hayek à prononcer en février 1931 quatre conférences à la London School of Economics. Celles-ci sont publiées en septembre 1931 sous la forme d'un ouvrage, Prix et Production. Dans ce contexte, la controverse est lancée au cours de l'été 1931 par Hayek qui publie dans le numéro d'août 1931 d'Economica la première partie d'une longue critique du Traité sur la monnaie. Keynes, très fâché ${ }^{1}$, répond dans le numéro de novembre 1931 d'Economica. La seconde partie de la critique est publiée par Hayek en février 1932.

Cet échange conduit à une correspondance entre Hayek et Keynes de décembre 1931 à mars 1932, dont il nous reste 12 lettres incluant une

* L'auteur remercie Michel Bellet et trois rapporteurs pour leurs remarques qui ont permis d'améliorer la version finale de l'article. L'auteur reste seul responsable des incompréhensions et erreurs.

** Université de Saint-Étienne, 6, rue Basse des Rives, 42023 Saint-Étienne. Tél: 04-77-42-19-61. Fax:0477-42-19-50. Email : zouache@univ-st-etienne.ir

1 " Hayek n'a pas lu mon livre avec la bonne volonté qu'un auteur est en droit d'attendre d'un lecteur. (...). II y a dans ses écrits une dimension passionnelle qui l'a conduit à s'en prendre à moi, sans qu'il me soit possible d'en déterminer l'origine. " (Keynes (1973), vol.13, p.243). 
lettre de Hayek à Keynes le jour de Noël et une réponse de Keynes le même jour (Keynes (1973), vol.13, pp.257-266). Le 29 mars 1932, Keynes met un point final à la discussion en annonçant à Hayek qu'il ne répondra pas à la seconde partie de la critique dans la mesure où il préfère consacrer son temps à « reformuler » sa théorie, une évolution qui conduira en 1936 à la Théorie Générale de l'emploi, de l'intérêt, et de la monnaie ${ }^{2}$. Hayek, qui avait dépensé beaucoup d'énergie à la préparation de ses critiques, est très déçu de la réaction de Keynes (Hayek (1966), p.241). Malgré l'envoi par Keynes d'un exemplaire de la Théorie Générale avant sa publication, Hayek n'en fera pas de recensions, une décision qu'il regrettera par la suite.

Si les échanges directs entre Hayek et Keynes cessent, la controverse prend une autre tournure en faisant appel aux membres des « cercles d'influence » des deux auteurs. Robinson (1933) propose de clarifier les concepts d'épargne et d'investissement du Traité. Mais c'est la recension de Sraffa en mars 1932 dans l'Economic Journal qui constitue l'attaque la plus virulente de la théorie de Hayek. Cette critique sera suivie d'un échange entre Hayek et Sraffa dans le numéro de juin ${ }^{3}$.

Le débat entre Hayek et Keynes a fait l'objet de nombreuses interprétations ${ }^{4}$. Selon Tieben (1994), l'origine de la controverse réside dans une incompréhension de chaque auteur vis-à-vis de la terminologie utilisée par l'autre. Steele (1993) propose quant à lui une comparaison des positions philosophiques des deux auteurs tandis que Dostaler ((1999), p. 763) considère que «c'est sur le plan éthique et politique que se situe le fossé entre les conceptions des deux hommes ». Notre interprétation insiste sur deux points trop souvent délaissés dans la littérature précédente ${ }^{5}$.

Premièrement, un des éléments clés du débat entre Hayek et Keynes repose sur deux conceptions alternatives de la coordination inter-individuelle. En effet, l'analyse des fluctuations conjoncturelles par Hayek et Keynes se caractérise par un point de départ commun, qualifié de question de la coordination: comment se coordonnent les actions des individus dans une économie décentralisée? De plus, Hayek et Keynes sont tous deux d'accord pour considérer que la coordination entre les agents ne se produit

2 “ Mais, sauf sur un point ou deux, qui peuvent être traités indépendamment du problème principal, je ne pense pas revenir à la charge dans Economica. J'essaie de reformuler et d'améliorer ma position centrale, et c'est probablement un meilleur moyen de passer son temps que de le consacrer à la controverse. " (Keynes (1973), vol.13, p.266).

3 Pour une analyse des échanges entre Sraffa et Hayek, voir Mongiovi (1990) et Lawlor et Horn (1992). D'après Lachmann ((1986), p.226), larticle de Sraffa fut d'une o férocité inhabituelle " par rapport aux recensions d'ouvrages usuelles de l'Economic Journal.

4 Voir notamment Hicks (1967), Dostaler (1991, 1999), Steele (1993), Tieben (1994), Cochran et Glahe (1994), Foss (1994), Caldwell (1998).

5 Afin d'offrir une perspective d'ensemble, la controverse entre Hayek et Keynes n'est pas seulement étudiée au regard des échanges parus dans Economica et l'Economic Journal. Nous tenons également compte des travaux de l'áconomiste autrichien relatifs notamment à l'organisation de la connaissance dans une économie de marché. De méme, notre analyse de la controverse délaisse le Traité dès la section 3 car la Thérie Générale constitue sur certains points une réponse aux critiques hayekiennes du Traité (Mongiovi, (1990)). Néanmoins, nous ne prenons pas en considération les travaux uitérieurs de Hayek, classés usuellement dans le champ de la philosophie politique el sociale (Hayek $(1978,1988)$ ). 
pas sans difficultés. Ils reconnaissent en cela l'éventualité d'un problème de coordination inter-individuelle. Néanmoins, Keynes et Hayek n'offrent pas une réponse similaire à la question de la coordination. La controverse entre Hayek et Keynes est par conséquent révélatrice de deux interprétations du problème de coordination:

- Le problème de coordination au sens de Hayek est la conséquence de chocs ou de mécanismes institutionnels exogènes - la monnaie et le crédit - qui perturbent les interactions entre les individus.

- Le problème de coordination au sens de Keynes est le résultat endogène des interactions entre les individus.

Deuxièmement, l'enjeu du débat Hayek-Keynes est de mettre en lumière l'existence d'une relation causale entre l'analyse de la coordination et la théorie de l'emploi. La nature du problème de coordination chez Keynes et Hayek conduit respectivement soit à la recherche des déterminants du chômage involontaire, soit à une théorie des fluctuations de l'emploi agrégé et du chômage temporaire.

Notre réflexion s'organise en quatre étapes. La deuxième section montre que les échanges entre Hayek et Keynes dans Economica et l'Economic Journal sont déjà influencés par la question de la coordination. Celle-ci désigne alors l'analyse des difficultés de coordination entre les décisions des épargnants et des investisseurs. La troisième section fait intervenir le rôle du concept d'équilibre général dans notre compréhension du problème de coordination. Le problème de coordination au sens de Hayek est tributaire de la notion d'équilibre intertemporel. Quant au problème de coordination keynésien, il ne peut pas être compris sans référence à la théorie générale de l'équilibre proposée par Keynes à partir du cadre analytique marshallien. La quatrième section compare les deux conceptions respectives du problème de coordination. Le problème de coordination au sens de Hayek résulte des erreurs d'anticipations que commettent les agents suite à un signal erroné. Les défauts de coordination peuvent être dépassés grâce à l'intervention de deux mécanismes de coordination parfaite, à savoir le système de prix et la concurrence. Dans l'appareil analytique keynésien, de tels mécanismes institutionnels ne permettent pas de coordonner efficacement les ressources. La théorie de la demande effective est alors l'expression de l'impossibilité de coordonner parfaitement les individus. En effet, le problème de coordination au sens de Keynes suppose que les agents forment des anticipations qui ne conduisent pas à un niveau de production de plein emploi. Enfin, la cinquième section étudie les implications du problème de coordination sur la théorie de l'emploi agrégé. S'agit-il d'une analyse des fluctuations de l'emploi et du chômage temporaire ou d'une théorie des déterminants des échecs de coordination à grande échelle, dont le plus marquant serait l'existence d'un grand nombre d'individus non employés au salaire existant dans une société et à une époque données? 


\section{Controverse Hayek-Keynes et coordination investissement/épargne}

Dans les premiers échanges entre Hayek et Keynes, la question de la coordination renvoie surtout aux problèmes de coordination entre épargnants et investisseurs ${ }^{6}$. Hayek et Keynes réalisent tous deux que les épargnants et les investisseurs sont des individus différents dont les décisions et les actions sont indépendantes les unes des autres de sorte que la coordination entre l'épargne et l'investissement ne se produit pas sans difficultés. Dans un premier point, nous présentons respectivement l'analyse de Keynes dans le Traité puis la critique de Hayek. Le deuxième point précise la réponse de Keynes concernant la coordination entre l'investissement (I) et l'épargne (S).

\subsection{Coordination I/S : du Traité à la critique de Hayek}

Dans le Traité, Keynes (1930) utilise ce qu'il nomme les équations fondamentales, des identités statiques à partir desquelles il reformule la théorie de Wicksell en termes d'écarts entre épargne et investissement. Lorsque l'épargne est supérieure à l'investissement, les entrepreneurs subissent des pertes, les prix diminuent et l'économie entre en récession. Inversement, quand l'investissement est supérieur à l'épargne, les entrepreneurs font des profits et l'économie entre dans une phase de boom. Par conséquent, dans le Traité, l'écart des profits par rapport à leur valeur nulle (ou profits d'aubaine) constitue la force principale du changement puisqu'il influence les niveaux de production des firmes et leurs demandes de facteurs. Lorsque les profits sont nuls, $I=S$, le système est en équilibre, c'est-à-dire qu'il se trouve dans un état stationnaire puisque les entrepreneurs ne sont pas incités à modifier leur échelle de production et le niveau d'emploi.

Mais, d'après Hayek, la théorie du cycle de Keynes ignore complètement la base de la théorie de Wicksell.

Tout d'abord, la critique de Hayek est fondée sur une définition de l'épargne comme consommation différée. Un acte d'épargne aujourd'hui signifie un désir de consommation demain. Une augmentation de l'épargne des ménages correspond à une consommation future plus élevée et nécessite donc un accroissement de l'investissement en biens de production. En effet, lorsque les agents décident de reporter leur consommation à une période ultérieure, la demande en biens de production augmente et la structure de production s'allonge; l'économie devient plus capitalistique. L'effet ultérieur sera un accroissement de la production finale de biens de consommation. Le

\footnotetext{
6 Nous ne proposons pas une lecture exhaustive des échanges entre Hayek et Keynes. En nous concentrant sur la question de la coordination des décisions d'épargne et d'investissement, nous ne traitons pas des discussions terminologiques relatives à la signification des différents concepts, en particulier le capital et les profits.
} 
temps de détour de la structure de production coïncide ainsi avec le décalage temporel de l'utilité des ménages.

En ce qui concerne le processus d'investissement, la critique réside dans l'idée que la définition de l'investissement de Keynes en tant que variable globale ne permet pas de tenir compte des variations de l'investissement entre les différents stades de production (Hayek (1931d), p. 274). La conséquence est d'oublier sous quelles conditions d'utilisation complète des ressources l'investissement et la consommation varient de façon inverse dans le court terme (Hayek (1931d), p. 286). Une hausse du prix des biens de consommation par rapport au prix des biens d'investissement provoque un accroissement de la production des biens de consommation et une diminution de la production des biens d'investissement. Si tous les facteurs de production sont utilisés, les ressources nécessaires à l'expansion de la production des biens de consommation proviennent du secteur des biens d'investissement. Les deux secteurs ne peuvent être tous deux en expansion en même temps.

\subsection{La réponse de Keynes : du Traité à la Théorie Générale}

La réponse de Keynes se comprend en deux étapes. Tout d'abord, ainsi que l'écrit Keynes ((1931b), p. 394), une grande partie de sa réponse peut être lue comme une recension critique de Prix et Production. Keynes accuse ensuite Hayek de se concentrer sur des problèmes terminologiques sans expliquer pourquoi les conclusions du Traité sont fausses. Selon Keynes, la nature de l'ajustement entre l'investissement et l'épargne constitue le coeur du désaccord avec Hayek.

Hayek souligne l'ambiguïté de la référence de Keynes à Wicksell concernant le concept de taux naturel d'intérêt. En effet, le taux naturel d'intérêt de Wicksell est un taux réel déterminé par l'offre et la demande relative de biens de consommation et de production. En revanche, le taux naturel de Keynes correspond au taux d'équilibre temporaire de l'épargne et de l'investissement et n'a pas les propriétés stabilisatrices du taux de long terme auquel songe Hayek. Finalement, Keynes répond à Hayek qu'il n'existe aucune théorie satisfaisante du taux d'intérêt réel de longue période (Keynes, (1931b), p. 394).

Il nous semble que, dans le Traité, Keynes développe une théorie du taux d'intérêt expliquant pourquoi un problème de coordination peut apparaître. Cette théorie diffère de celle de Hayek dans la mesure où elle considère comment les prix des actifs affectent les flux d'épargne et d'investissement (Keynes (1931b), pp. 390-91). Dans ses premiers écrits, Hayek identifie simplement ces flux respectifs avec l'offre et la demande de fonds prêtables. En revanche, Keynes affirme que l'épargne ne constitue pas automatiquement une offre de fonds prêtables. Il distingue la décision d'épargner des ressources courantes et la décision de prêter une épargne aux investis- 
seurs. Keynes réalise, par rapport à Hayek, que les ménages ont le choix entre une variété d'actifs financiers pour déterminer la forme sous laquelle l'épargne dirige la consommation future. La demande et l'offre de fonds prêtables ne sont pas autonomes mais sont influencées par les opérations sur les autres marchés. Keynes affirme que, indépendamment des actions des autorités monétaires, les marchés financiers transmettent les perturbations provoquées par les comportements spéculatifs. Ainsi, la quantité de monnaie peut varier sans effet sur l'épargne et l'investissement car il existe une sphère financière autonome.

Keynes reconnaît néanmoins les faiblesses de son ouvrage concernant la théorie du capital. La critique de Hayek relative à l'absence de théorie du capital dans le Traité a exercé une influence sur Keynes, notamment lorsqu'il introduit les concepts de préférence pour la liquidité et d'efficacité marginale du capital dans la Théorie Générale ${ }^{7}$. Keynes propose également une analyse différente de la coordination entre l'investissement et l'épargne fondée sur la théorie de la demande effective qui apparaît comme l'expression d'un raisonnement en équilibre général.

En effet, la coordination entre les épargnants et les investisseurs s'effectue dans le cadre d'une économie en équilibre général. Il semble par conséquent important de rechercher dans quelle mesure le problème de coordination inter-individuelle est dépendant du concept d'équilibre général auquel Keynes et Hayek se réfèrent.

\section{Théorie de l'équilibre général et problème de coordination}

Quel est le rapport entre le problème de coordination inter-individuelle et la référence à une conception particulière de l'équilibre général ? L'objectif de la théorie hayekienne des cycles consiste à dépasser l'équilibre général walrasien en intégrant les fondements autrichiens de la théorie du capital. Hayek propose ainsi une analyse de la coordination intertemporelle des décisions des agents économiques. Quant à Keynes, la volonté de dépasser l'analyse partielle marshallienne le conduit à un problème de coordination insistant sur les interactions entre des individus évoluant au sein de marchés interreliés mais fonctionnant de manière autonome.

\footnotetext{
7 “ Je suis d'accord avec lui [Hayek] pour dire qu'une explication claire des tacteurs déterminant le taux naturel d'intérêt devrait avoir une place dans un Trailé sur la Monnaie complet, et qu'efle est absente dans le mien (...). Plus tard, je m'elforcerai de combler cette déficience. " (Keynes, (1931b), pp. 394-95)
} 


\section{1 Équilibre général walrasien et problème de coordination au sens de Hayek}

Nous ne traitons pas de la filiation entre Walras et Hayek du point de vue de l'équilibre général, une filiation qui mériterait d'être discutée de façon plus approfondie ${ }^{8}$. Notre raisonnement s'inspire de la théorie hayekienne des cycles d'affaires qui semble plus ancrée dans la théorie de l'équilibre général walrasien (Hicks (1982), Lachmann (1986)) ${ }^{9}$. En outre, d'après Hayek, le cycle ne peut être analysé qu'en prenant pour référence une situation d'équilibre général walrasien :

«L'introduction des phénomènes cycliques au sein de la théorie économique de l'équilibre, avec lequel ils sont en contradiction apparente, reste le problème crucial de la théorie des cycles d'affaires. " (Hayek (1933a), p. 33).

Comment résoudre cette contradiction entre la référence à un concept - l'équilibre général walrasien - se produisant dans le temps logique et une analyse dont l'ambition est d'expliquer la dynamique intertemporelle des économies? En d'autres termes, comment mettre en évidence un mode de coordination susceptible de faire apparaître des fluctuations économiques à partir d'une référence à l'équilibre général walrasien? Afin de répondre à ces questions, Hayek propose une analyse de la coordination fondée sur la notion d'équilibre intertemporel. Ce concept permet tout d'abord de comprendre les conditions de la réalisation d'une coordination parfaite des décisions et actions des individus. L'écart par rapport à l'équilibre intertemporel traduit ensuite l'existence d'un problème de coordination inter-individuelle.

«Toute activité économique est conduite à travers le temps », telle est la première phrase de l'article de 1928, "Intertemporal Price Equilibrium and Movements in the Value of Money", dont l'objectif affiché est d'intégrer le temps au sein de la théorie de l'équilibre général walrasien. Le temps est conçu comme une succession ordonnée de points reflétant une technique et une préférence données. Les conditions de réalisation d'un équilibre intertemporel sont parallèles aux conditions de réalisation d'un équilibre statique. La «seule» différence est que les biens sont situés à différents moments du temps, même s'ils sont techniquement équivalents : des biens techniquement identiques ont, suite à leur disponibilité temporelle, des prix

8 Pour une telle discussion, voir Butos (1985), Colonna (1990). Des différences existent entre l'approche de Walras et celle de Hayek. Ainsi, la dimension temporelle est très marquée dans la théorie hayekienne des cycles relativement à l'analyse de Walras. Notons également que la référence à l'équilibre général walrasien devient beaucoup moins évidente à mesure de l'évolution de l'oeuvre de Hayek. En particulier, les articles de 1937 et 1945 sur la connaissance dispersée et le processus de concurrence adoptent une position beaucoup plus critique vis-à-vis de l'équilibre général walrasien.

9 La théorie hayekienne des fluctuations conjoncturelles est généralement associée à deux ouvrages majeurs. Monetary Theory and the Trade Cycle (1933a) s'intéresse beaucoup plus aux facteurs monétaires qui causent le cycle tandis que Prices and Production (193ic) est principalement consacré au phénomène réel qui constitue le cycle. Hayek approfondit son analyse dans une série d'articles regroupés dans Profits, Interest and Investment (1939). Certaines idées sont développées dans plusieurs articles des années vingt, rassemblés par McCloughry (1984), en particulier "Intertemporal Price Equilibrium and Movements in the Value of Money" (1928). Des versions parfois plus complètes et d'autres textes des années vingt sont regroupés dans le volume 5 des Collected Works de Hayek. 
différents. Du fait de la difficulté de transfert temporel des marchandises, le rapport d'échange entre deux biens évolue entre deux périodes.

L'équilibre intertemporel désigne une norme à partir de laquelle il est possible de comprendre l'analyse hayekienne du problème de coordination inter-individuelle. Sur le sentier d'équilibre intertemporel, la structure de production est en équilibre. Les entrepreneurs produisent pour satisfaire la demande des consommateurs de sorte que l'égalité entre offre et demande est satisfaite aussi bien dans le secteur des biens de production que dans le secteur des biens de consommation. Par conséquent, les décisions d'investissement des entrepreneurs correspondent aux intentions d'épargne des ménages. À l'équilibre intertemporel, les décisions des individus, en particulier celles des épargnants et des investisseurs, sont donc parfaitement coordonnées.

Un problème de coordination apparait lorsque l'introduction de la monnaie sous forme de crédit perturbe les conditions d'équilibre intertemporel en modifiant l'égalité entre épargne et investissement. Le crédit entraîne une extension disproportionnée des détours de production et perturbe le mécanisme régulateur des prix relatifs. L'adaptation des entrepreneurs à cette nouvelle situation rompt l'équilibre de la structure de production, et l'économie s'écarte de son sentier d'équilibre intertemporel.

\subsection{La Théorie Générale: une « généralisation 》 de l'équilibre partiel marshallien}

Malgré une certaine continuité analytique entre le Traité et la Théorie Générale (Leijonhufvud, (1981)), il semble que le Keynes de 1936 soit beaucoup plus imprégné d'une influence marshallienne que wicksellienne. L'analyse de la coordination entre l'investissement et l'épargne est alors conduite en référence à la théorie de la demande effective. L'idée fondamentale est que le produit et l'emploi sont contraints par la demande agrégée des biens et non par l'offre des travailleurs ou la capacité de l'économie à produire des biens ou des services (Tobin (1997)). Or, le principe de demande effective relève d'une démarche macroéconomique en équilibre général.

En effet, le système économique chez Keynes est composé de plusieurs marchés en équilibre partiel. Keynes (1936) propose un raisonnement en équilibre général lorsqu'il étudie les interdépendances entre les différents marchés marshalliens afin de construire une théorie de l'emploi. La démarche est fondée sur une méthodologie de l'équilibre général puisque le fonctionnement d'un marché particulier dépend de l'état d'un ou de plusieurs marchés ou même de la situation économique dans son ensemble. Le niveau d'emploi est ainsi déterminé par le montant produit sur le marché des biens. Mais, la production globale est elle-même fonction de la demande effective, dont le montant dépend des revenus versés aux salariés. Ce réseau complexe d'interactions macroéconomiques forme le cadre fondamental de la théorie 
générale de l'équilibre de Keynes dont le principe de demande effective est l'expression ultime.

Le principe de demande effective introduit une distinction entre une composante autonome de la demande et une composante induite par le revenu qui la finance, et qui dépend du niveau de l'emploi. Celui-ci correspond à l'emploi créé par l'entreprise à laquelle s'adresse la demande, mais aussi et surtout à l'emploi créé par d'autres entreprises, essentiellement dans d'autres branches. Une telle analyse fait introduire l'interdépendance entre les marchés des facteurs et les marchés des produits, et nécessite ainsi le recours à une démarche macroéconomique. On est alors en présence d'une boucle de rétroaction qui relève typiquement d'un raisonnement en équilibre général. Les entrepreneurs décident du montant produit en fonction de la demande anticipée. Ils distribuent un revenu à leurs salariés qui forme le socle de la demande future.

L'examen du problème de coordination chez Keynes (1936) s'opère alors dans le cadre d'une théorie générale de l'équilibre marshallien ${ }^{10}$. Plusieurs travaux ont mis en évidence que Keynes propose une « généralisation » de la méthodologie marshallienne (Clower (1989), De Vroey (1999a,b)). On parle de «connexion marshallienne » (Clower (1989)) dans la mesure où la théorie de l'équilibre général chez Keynes consiste à appliquer la méthode d'équilibre partiel de Marshall de confrontation entre prix d'offre et prix de demande au système pris dans son ensemble, et non plus aux firmes ou industries représentatives :

«sa [Alfred Marshall] théorie du produit et de la consommation dans son ensemble, par opposition à sa théorie de la production et de la répartition d'un produit donné, ne fut jamais exposée. " (Keynes, 1973, vol. 7, p. 29).

A partir des fondements marshalliens, il est alors possible d'étendre l'analyse de courte période d'un marché particulier au cas d'une production globale.

Dans sa construction analytique, la théorie de la demande effective est ainsi fondamentalement marshallienne. Elle désigne le montant du produit attendu au point d'intersection entre la courbe de la demande globale et la courbe d'offre globale. En ce sens, la théorie de la demande effective détermine plutôt un point de demande effective, c'est-à-dire le point où la demande devient effective et qui provoque généralement du sous-emploi et une sous-utilisation des capacités de production (Pasinetti (1997)). Le point d'intersection de la courbe de demande globale et de la courbe d'offre globale détermine le volume de l'emploi car, à ce point, la prévision de profit des entrepreneurs est maximum. Le principe de la demande effective montre que la demande n'est pas indépendante de la quantité produite puisqu'elle est financée par les revenus qui en sont issus. Or, la méthode de l'analyse partielle marshallienne néglige cet effet. Le passage du cadre marshallien d'analyse partielle à la théorie générale de l'équilibre de Keynes se fait ainsi par l'endogénéisation du revenu.

10 Pour une compréhension plus approtondie de l'analyse de Marshall, voir De Vroey (1999a,b). 
Le problème de coordination peut dès lors se comprendre à partir de l'observation suivante : si la demande effective détermine la quantité de facteurs employée sur le marché du travail, les revenus versés déterminent la demande effective. Mais, les déficiences de la demande effective signifient que le montant du produit attendu par les entrepreneurs est inférieur au niveau de plein emploi. Ainsi, la généralisation de l'analyse partielle marshallienne montre que les difficultés de coordination des décisions individuelles s'expriment au niveau de l'équilibre général de l'économie. Le problème réside dans la nécessaire coexistence de certains comportements individuels qui doivent être non seulement cohérents avec le comportement des autres individus mais aussi mutuellement compatibles au sens de l'équilibre général.

\section{Une analyse du problème de coordination : erreur ou échec de coordination?}

Le problème de coordination intervient-il comme un élément exogène ou endogène dans le système analytique des deux auteurs? Dans le cas d'une réponse positive, le problème de coordination est qualifié de temporaire. $\mathrm{Si}$ la réponse est négative, le problème de coordination est dit permanent; et par conséquent inhérent aux économies décentralisées. La problématique de la coordination est considérée à travers deux perspectives opposées.

Dans la théorie hayekienne, seules des perturbations exogènes, de nature informationnelle, provoquent une déviation temporaire par rapport à l'équilibre intertemporel. Les échecs de coordination sont le résultat d'une erreur ou plutôt d'une multitude d'erreurs provenant de l'existence d'un signal erroné. En revanche, chez Keynes, les interactions entre les individus sont la source d'une instabilité intrinsèque des économies de marché. Il n'est alors pas possible d'éliminer totalement les difficultés de coordination inter-individuelle.

\subsection{Une analyse du problème de coordination au sens de Hayek}

Les travaux ultérieurs de Hayek ${ }^{11}$ mettent à jour sa position fondamentale quant à la conception de la coordination des individus au sein d'une économie décentralisée. L'éventualité d'un problème de coordination naît de l'hypothèse d'une connaissance dispersée. Cependant, la théorie de Hayek ne tire pas les conséquences analytiques du problème de coordination interindividuelle puisqu'il existe une institution capable d'allouer de manière efficiente les ressources : le système de prix.

11 Avec notamment "Economics and Knowledge" (1937), Profils, Interest and Investment (1939), "The Use of Knowledge in Society" (1945). 
La question de la coordination consiste à savoir (Hayek (1937), p. 50) :

" comment l'interaction spontanée d'un grand nombre d'agents, chacun possédant seulement des fragments de connaissance, provoque un état des affaires dans lequel les prix correspondent aux coûts, etc., et qui pourrait seulement être obtenu par l'action délibérée d'un agent possédant la connaissance de l'ensemble des individus. "

Le problème de la connaissance dispersée est ainsi fortement tributaire de la communication de l'information supplémentaire dont l'agent a besoin afin que ses décisions s'insèrent dans le système économique. Ce point est décisif dans l'analyse hayekienne des cycles, notamment telle qu'elle est exposée dans Profits, Interest and Investment (1939). En particulier, "Price Expectations, Monetary Disturbances and Malinvestment" (1933b), qui répond à la critique de Prix et Production par Myrdal, nous permet de faire le lien entre les premiers travaux de Hayek sur les cycles et ses articles sur la dispersion de la connaissance.

Dans cette perspective, les fluctuations économiques sont indissociables de la division de la connaissance. Les entrepreneurs, faute d'informations adéquates, ne peuvent prévoir les plans de consommation et d'épargne des ménages. Ils fondent alors leurs plans de décision en fonction de l'information fournie par le système bancaire - l'expansion du crédit - qui correspond dans la théorie hayekienne à un faux signal. Les erreurs d'anticipation qui en résultent provoquent de faux investissements ("malinvestment") à l'origine d'une déformation temporaire de la structure de production.

Cependant, si Hayek souligne effectivement l'importance des difficultés de coordination inter-individuelle dans une économie décentralisée, c'est pour mieux identifier les mécanismes assurant une allocation optimale des ressources. L'efficience n'est pas définie en utilisant le critère de $\mathrm{Pa}$ reto. Elle concerne la capacité d'une institution donnée à transformer la connaissance subjective et dispersée en information objective et uniforme. Or, le marché dispose d'un mécanisme institutionnel à fort pouvoir coordinateur, la concurrence, dont résulte un signal à fort contenu d'information et synthétisant la connaissance dispersée, le système de prix.

\section{Le système de prix comme mécanisme de coordination}

Les prix apparaissent comme des indices au sein desquels l'information pertinente et dispersée est concentrée. Ils permettent aux agents d'utiliser une connaissance dont ils sont ignorants. Le système de prix coordonne les actions d'agents différents dans un monde où la connaissance est fragmentée. En fait, en tant que principe de coordination, les prix permettent la convergence de l'information dispersée vers une information unique : cette convergence forme le processus de marché.

Parmi les prix les plus significatifs, c'est-à-dire contenant l'information indispensable à la coordination, le taux d'intérêt naturel et le prix relatif des biens de consommation et des biens de production sont les plus importants. 
En effet, le taux d'intérêt naturel révèle la rentabilité marginale future de tout investissement nouveau. Les entrepreneurs ne doivent pas considérer le taux d'intérêt de marché et la quantité de crédits distribués pour décider de leurs plans d'investissement. Sinon, leurs prévisions risquent d'être déçues. De plus, le prix relatif des biens de consommation par rapport aux biens de production indique les préférences des individus en termes de consommation et d'épargne. De même, les entrepreneurs commettent des erreurs s'ils considèrent que le taux d'intérêt de marché leur procure l'information suffisante concernant les choix intertemporels de consommation des agents économiques. Finalement, seul le taux d'intérêt de marché constitue le signal de prix erroné. On comprend vite pourquoi : dans l'analyse de Hayek, il n'est pas le résultat de la libre concurrence sur le marché mais il est fixé de manière arbitraire par les banques. Il ne contient donc aucune information pertinente, soit de la situation économique, soit des préférences des agents.

\section{La concurrence comme procédure de coordination}

Dans une société complexe, surviennent d'instant en instant des évènements ne figurant pas dans les données des agents. Le processus d'équilibration sur le marché nécessite une explication du mécanisme par lequel les données sont ainsi ajustées. C'est le système de communication constitué par les prix sur un marché libre qui, en transmettant continuellement des informations sur les changements survenus, conduit les acteurs à changer leurs plans. Or, ce système fonctionne à la condition que les individus soient libres de modifier leurs comportements, ce qui se produit par le processus effectif de concurrence. Seule la concurrence permet la rencontre des connaissances dispersées. La concurrence est une procédure de découverte de faits nouveaux, c'est-àdire qui ne faisaient pas partie des données initiales du plan (Hayek (1978)). Chaque agent est en mesure de faire évoluer ses plans en fonction des opportunités qui se présentent. La concurrence est conçue comme un processus d'ajustement mutuel dans la mesure où le processus de marché fonctionne comme une succession d'étapes où les agents adaptent leurs plans suite aux erreurs commises.

Ainsi, le problème de la coordination s'inscrit dans une problématique d'erreurs d'interprétation. Chez Hayek, si les signaux de prix sont pertinents et contiennent toute l'information disponible, le problème de la coordination inter-individuelle ne subsistera pas. En revanche, Keynes ne peut s'en tenir à une constatation des difficultés temporaires de la coordination des individus dans une économie décentralisée.

\subsection{Une analyse du problème de coordination au sens de Keynes}

Il est difficile, voire prétentieux, de séparer clairement les différents éléments constitutifs du problème de coordination tant l'analyse de Keynes est com- 
plexe. Aussi, nous ne présenterons pas complètement la pensée de Keynes ${ }^{12}$. La thèse défendue dans cette section est que les échecs de coordination macroéconomique développés dans la Théorie Générale sont la conséquence directe du problème de coordination inter-individuelle. En effet, même si les effets des défauts de coordination s'exercent au niveau macroéconomique les revenus distribués par les producteurs ne provoquent pas un montant de demande effective suffisant au plein-emploi des ressources en travail le principe de demande effective exprime les difficultés de coordination des anticipations des agents.

En ce sens, il existe une différence entre le Traité et la Théorie Générale quant au problème de coordination. Si, dans le Traité, Keynes ((1930), p. 143) précise que ce sont les profits ou pertes anticipés plutôt que les profits ou pertes actuels qui influencent les décisions d'investissement des entrepreneurs, il ne développe pas une analyse approfondie du rôle des anticipations dans le problème de coordination. Ainsi que Keynes ((1936), p. 101) le précise, le Traité n'établit pas de distinction claire entre les résultats attendus et les résultats réalisés. Avec le principe de demande effective apparaît le rôle crucial des anticipations dans le problème de coordination au sens de Keynes.

En effet, si on se situe en courte période (technologie et équipements fixés, taux de salaire monétaire pré-déterminé), ce sont les anticipations sur les conditions de demande qui joue un rôle actif dans le processus de décision des entrepreneurs :

« Tout producteur doit essayer d'estimer la demande qui a des chances d'être effective avant de débuter toute production et, ce, indépendamment de la capacité productive courante. Ne pas tenir compte de cet aspect provoque tout simplement un «engorgement du marché ». En ce sens, à tout point du temps, c'est la demande anticipée (la demande effective de Keynes) qui détermine le niveau de production. " (Pasinetti (1997), pp.100-101).

L'importance des anticipations des agents se comprend très bien lorsque l'on se penche sur les difficultés de coordination des décisions d'épargne et d'investissement.

D'après Keynes, un acte d'épargne signifie une réduction nette de la demande, c'est-à-dire de la demande d'utilisation des ressources telle qu'elle est perçue par les producteurs. Par rapport à Hayek, l'acte d'épargner ne constitue pas un signal indiquant quand et où les ressources doivent être transformées en biens de consommation. ll manque des marchés futurs où la demande accrue de produits spécifiques serait communiquée aux producteurs respectifs.

De plus, l'épargne ne constitue pas seulement un stock de monnaie utilisé à des fins d'investissement physique. L'épargne est également constituée d'actifs financiers échangés sur le marché des titres. Or, la sphère

12 Nous insistons sur les problèmes de coordination intertemporelle des décisions des consommateurs, des producteurs, des investisseurs et des spéculateurs. En privilégiant cette perspective, nous omettons le rôle de la monnaie dans le problème de coordination. Pour une étude plus poussée de l'importance de la monnaie dans l'analyse des défauts de coordination chez Keynes, voir Dos Santos Ferreira (1999). 
financière peut très bien fonctionner indépendamment de la sphère réelle. L'évolution de l'épargne sur le marché des titres reflète alors les variations des anticipations des spéculateurs. Keynes indique que ces anticipations sont très volatiles.

En effet, Keynes insiste sur l'impossibilité de prévoir parfaitement les actions d'individus dont le comportement est sujet à des « esprits animaux ». Le comportement des entrepreneurs est affecté par des mouvements sans fondements rationnels qui expliquent, au moins en partie, la variabilité de l'investissement. Le marché financier facilite la mobilisation du capital en fournissant des liquidités aux investisseurs individuels qui, par son intermédiaire, convertissent à tout instant des titres en monnaie. Cependant, un effondrement brutal des prévisions à long terme est possible, ce qui conduit les principaux détenteurs de titres à se précipiter pour liquider leurs positions. La perte de confiance dans les revenus futurs des actifs en capital a des effets sur la formation des capitaux physiques, favorisant la formation d'un chômage massif.

Le problème de coordination inter-individuelle apparaît comme la conséquence du degré d'horizon différent des décisions d'agents autonomes. Dans le financement de l'investissement, on constate un décalage entre le processus de formation des anticipations des producteurs (horizon de long terme) et le processus de formation des anticipations des spéculateurs (horizon de court terme). En ce sens, le taux d'intérêt ne joue pas son rôle de coordination des décisions et ne permet pas d'assurer un financement de l'investissement correspondant au plein emploi des facteurs de production.

Ainsi, les individus prennent leur décision à partir d'anticipations sur le comportement des autres agents, intervenant soit sur le même marché, soit sur d'autres marchés. Or, il est difficile pour un entrepreneur, lorsqu'il prend une décision sur le marché des biens, d'anticiper parfaitement à la fois le niveau produit par les autres entrepreneurs et les revenus des consommateurs. Un problème de coordination apparait du fait du caractère intrinsèquement imparfait des décisions prises. En effet, les entrepreneurs ne peuvent tenir compte des externalités de la demande. Ils ne comprennent pas que les salaires qu'ils versent se traduisent par une consommation de leurs propres produits et surtout des produits des autres entrepreneurs. Si chaque producteur prenait en considération cet effet, il serait incité à verser des salaires plus élevés, à produire plus, et, par suite, à employer un plus grand nombre d'individus.

Les défauts de coordination sont la conséquence de l'incapacité des individus à percevoir correctement et complètement les opportunités issues des décisions et actions des autres agents économiques. Dans cette perspective, le problème de coordination au sens de Keynes signifie que les individus forment des anticipations qui ne correspondent pas à un niveau de production de plein emploi. En d'autres termes, le problème de coordination au sens de Keynes suppose que les attentes sont réalisées mais que les prévisions ne sont pas parfaites au sens où elles ne permettent pas d'atteindre 
un niveau de production de plein emploi. En revanche, le problème de coordination au sens de Hayek implique que les entrepreneurs commettent des erreurs, ce qui n'est pas le cas dans l'analyse de Keynes.

En outre, le principe de demande effective témoigne de la différence fondamentale entre Keynes et Hayek concernant le rôle des prix comme mécanisme de coordination entre les individus. Dans l'analyse hayekienne, le système de prix permet de résoudre le problème de coordination interindividuelle. Dans la théorie de Keynes, les prix ne constituent pas un signal de coordination parfaite puisque, par exemple, les décisions d'épargne et d'investissement, ou l'offre et la demande de travail, ne sont pas déterminées par les mêmes variables. Les prix ne synthétisent pas l'ensemble de l'information dont les entrepreneurs ont besoin lors de leur décision de production.

Les implications sur la conception du chômage sont étudiées à la section suivante. Dans le premier cas, les erreurs provisoires conduisent à un chômage temporaire. Dans le second cas, le chômage est un phénomène révélant l'incapacité des individus à coordonner leurs décisions. Il est alors dit involontaire au sens de Keynes.

\section{$5 \quad$ Mode de coordination et théorie de l'emploi}

L'intérêt du débat Hayek-Keynes est également de révéler l'importance du problème de coordination dans une théorie de l'emploi agrégé. Au sein du cadre analytique hayekien, la déformation de la structure de production provoque une réallocation de la force de travail, à l'origine d'un chômage temporaire. En revanche, le caractère permanent du problème de coordination inter-individuelle chez Keynes rend possible la démonstration de l'existence d'un nombre important d'individus non employés au salaire existant dans une économie donnée.

\subsection{Problème de coordination, fluctuations de l'emploi et chômage temporaire chez Hayek}

La coordination intertemporelle autrichienne fondée sur l'équilibre général walrasien est à l'origine d'une analyse des fluctuations de la production conçues comme la conséquence de la modification de l'allocation des ressources, et notamment du facteur travail. En effet, si on désire expliquer les fluctuations de la production,

" nous devons commencer à l'endroit où s'arrête la théorie économique générale, c'est-à-dire à partir d'une condition d'équilibre de plein emploi de 
toutes les ressources. » (Hayek (1931c), p. 97) ${ }^{13}$

Deux conséquences sont alors importantes pour notre propos.

Tout d'abord, que déduire de cette position par rapport à l'explication des fluctuations proposée par Hayek? Si le point de départ de l'analyse est une situation où toutes les ressources en facteurs de production sont employées, l'accroissement du produit dans le cas d'une augmentation de l'épargne volontaire des ménages se traduit par l'adoption de méthodes de production plus capitalistiques. L'organisation de la production est telle qu'un plus grand nombre d'individus est employé dans le secteur des biens d'investissement au détriment des biens de consommation. Mais, puisque toutes les ressources en facteurs de production sont utilisées, il n'est pas possible d'envisager un accroissement absolu de l'emploi. Seule s'est produite une nouvelle allocation du facteur travail. Le raisonnement ne peut être que parallèle concernant le cas opposé de la dépression. La période de crise se traduit dans cette logique par une ré-allocation du travail vers le secteur des biens de consommation et non par l'apparition d'un chômage.

Pourtant, d'après Hayek, seule cette conception permet de faire apparaître une situation dans laquelle il est temporairement impossible d'employer toutes les ressources disponibles (Hayek (1931c), p. 100). Si on admet ce traitement asymétrique, c'est-à-dire qu'il n'est pas possible d'accroître la quantité d'individus employés en situation de cycle ascendant puisque tous les facteurs de production sont utilisés mais qu'il est possible de diminuer l'emploi total en situation de cycle descendant, l'économiste autrichien ne peut envisager qu'un chômage temporaire défini comme une impossibilité momentanée d'utiliser toutes les ressources disponibles.

Le chômage temporaire est la conséquence de l'affectation erronée du montant donné de ressources suite au faux signal des autorités monétaires. Un nombre plus ou moins important d'individus (Hayek ne le précise pas) se trouve en situation précaire, en attente d'une nouvelle organisation de la production correspondant aux préférences d'épargne des ménages. Selon Hayek, la crise et le chômage, sont des phénomènes naturels qui se résorbent seuls afin de parvenir à un nouvel équilibre. La seule façon de lutter contre le chômage temporaire consiste à ne pas utiliser de stimulants artificiels - que ce soit au cours de la crise ou après - mais de laisser au temps le soin d'opérer une guérison durable par le lent processus d'adaptation de la structure de production aux moyens disponibles pour la formation du capital.

13 L'utilisation du terme " plein emploi » peut sembler discutable puisqu'il s'agit d'un concept typiquement keynésien. Nous utilisons ici la citation française (1975). À partir de la citation originale ((édition 1935), p. 34), ll est préférable de se référer à " une condition d'équilibre où toutes les ressources sont utilisées ":

"we have to start where general economic theory stops; that is to say at a condition of equilibrium when no unused resources exist." 


\subsection{Problème de coordination et théorie du chômage involontaire chez Keynes}

Malgré les précautions de Keynes relatives à la classification des différents types de chômage, la définition proposée du chômage involontaire est à l'origine de multiples confusions terminologiques mais également conceptuelles. La définition généralement admise est fondée sur la courbe d'offre de travail : le chômage est involontaire lorsque les travailleurs sont en dehors de leur courbe d'offre, de sorte qu'il existe un excès de l'offre de travail au salaire réel courant. Cependant, l'examen du problème de coordination au sens de Keynes proposé dans la section précédente détermine un concept de chômage involontaire résultant de défauts de coordination.

En effet, le chômage involontaire fonde le concept keynésien de sousemploi puisque le plein emploi est alors conçu comme l'absence de chômage involontaire (Dos Santos Ferreira (1999), p. 10). Or, Keynes considère que le plein emploi est atteint lorsque l'emploi global cesse de réagir élastiquement aux accroissements de la demande effective des produits qui en résultent (Keynes (1936), p. 54). La conception sous-jacente du chômage est alors étroitement dépendante du principe de demande effective. La théorie du chômage involontaire au sens de Keynes doit finalement être examinée dans cette perspective. Le chômage involontaire est alors le corollaire logique d'un niveau de la demande effective inférieur au niveau de plein emploi. En d'autres termes, la loi de Say, et son corollaire le plein emploi, ne sont pas vérifiés. Chômage involontaire, déficiences de la demande effective ou absence de plein emploi découlent d'un même constat analytique : il existe un problème de coordination inter-individuelle dans les économies décentralisées.

Supposons, dans une économie donnée, un niveau de production correspondant au montant de la demande effective. Le produit global dépend de la demande d'investissement et de la demande de consommation. La demande globale ainsi constituée ne correspond pas néanmoins à un montant de demande effective compatible avec la production de plein emploi. Des déficiences de demande effective impliquent l'existence d'un chômage involontaire. De plus, ce chômage contraint la demande : un processus cumulatif maintient une situation de chômage involontaire à un niveau éloigné du plein emploi.

Le chômage involontaire est en ce sens un concept d'équilibre général car il est dérivé d'une théorie générale des interrelations entre les marchés. Le chômage n'est pas un phénomène qui relève d'un problème de coordination sur un seul marché, à savoir le marché du travail. C'est pourquoi la rupture du principe du salaire de réservation est une condition nécessaire mais pas suffisante à la présence du chômage involontaire (Leijonhufvud (1998), p. 228). Keynes refuse en effet d'affecter l'explication du chômage involontaire à un comportement des salariés qui provoquerait des rigidités institutionnelles sur le marché du travail. Le test d'explication du chômage involontaire concerne l'incapacité d'un individu, étant donné le fonctionne- 
ment du marché du travail - et notamment étant donnée l'hypothèse de rigidité des salaires nominaux - à trouver un nouvel emploi même lorsqu'il accepte un salaire nominal plus faible que celui qui prévaut sur le marché.

Un individu donné ne peut en effet pas agir sur le fonctionnement du système économique caractérisé par des interactions non coordonnées d'agents évoluant au sein des marchés des biens, de la monnaie, des actifs et du travail. Ces interactions peuvent produire dans certaines conditions des échecs de demande effective, à l'origine d'un résultat non attendu, que Keynes nomme le chômage involontaire. Dans cette perspective, le théoricien du chômage ne doit pas s'intéresser à la volonté d'un individu de participer ou non au marché du travail mais plutôt à son incapacité à ne pas trouver un emploi. La théorie de Keynes intervient alors pour offrir un cadre analytique permettant de démontrer la possibilité d'une telle incapacité de l'individu, laquelle, lorsqu'elle est transférée au niveau de l'équilibre général, relève de l'impossibilité d'une économie décentralisée à coordonner efficacement les décisions individuelles.

\section{Conclusion}

L'interprétation proposée de la controverse Hayek-Keynes met en évidence la richesse d'une optique de lecture en terme de coordination. Le débat entre Hayek et Keynes atteste de l'intérêt d'une étude de la relation entre le problème de coordination inter-individuelle et la conception des fluctuations économiques.

La perspective autrichienne de la structure de production conduit Hayek à une théorie des cycles fondée sur l'équilibre général walrasien. L'apport de Hayek est de prendre au sérieux le fait qu'il peut exister un problème dans la coordination des individus en insistant notamment sur la dispersion de la connaissance dans une économie décentralisée. Mais, Hayek, en se référant à des mécanismes de coordination walrasien, en particulier l'existence d'un système de prix relatif et la concurrence, détermine les conditions de résolution de ce problème. Un tel cadre implique une étude des fluctuations de l'emploi et l'explication d'un chômage qualifié de temporaire. En revanche, Keynes envisage des défauts de coordination ne pouvant pas être éliminés par les informations disponibles dans une économie walrasienne. Seule l'analyse de Keynes conçoit un problème de coordination inter-individuelle permanent et démontre ainsi l'existence du chômage involontaire. La conceptualisation des défauts de coordination nécessite de considérer qu'ils sont le résultat des interactions entre des individus évoluant au sein de marchés interdépendants.

Hayek et Keynes fondent leur étude des fluctuations sur un problème de coordination des anticipations des individus. Malgré cette apparente similitude, les deux auteurs proposent une analyse sensiblement différente. 
La conception hayekienne correspond à une problématique d'erreurs d'anticipations. En ce sens, le problème de coordination ne peut-être envisagé sans l'existence d'une norme qui détermine les conditions d'une coordination parfaite des décisions et actions des individus. En revanche, le processus de formation des anticipations chez Keynes intègre en son sein les conditions endogènes de l'apparition d'un problème de coordination. Il est intéressant de noter que, même si les attentes des agents sont vérifiées, elles provoquent une situation de sous emploi des ressources, révélant ainsi la permanence d'un problème de coordination inter-individuelle dans les économies de marché.

\section{Références}

Butos, W. (1985), "Hayek and general equilibrium analysis", Southern Economic Journal, vol.52, $\mathrm{n}^{\circ} 2$, pp.332-343.

Caldwell, B. (éd.) (1995), Contra Keynes and cambridge, the collected works of F.A. Hayek, vol.9, London, Routledge.

Caldwell, B. (1998), "Why didn't Hayek review Keynes's general theory", History of Political Economy, vol.30, $n^{\circ} 4$, pp.545-569.

Clower, R. (1989), "Keynes' general theory: the Marshall connection", in D. Walker (éd.), Perspectives on the History of Economic Thought, Aldershot, Edward Elgar, vol. 2, pp. 133-147.

Cochran, J. et F. Glahe (1994), "The Keynes-Hayek debate:lessons for contemporary business cycle theorists", History of Political Economy, vol.26, $\mathrm{n}^{\circ} 1$, pp.69-94.

Colonna, M. (1990), "Hayek on money and equilibrium", Contributions to Political Economy, vol.9, pp.43-68.

De Vroey, M. (1999a), "Keynes and the Marshall-Walras divide", Journal of the History of Economic Thought, vol.21, n'2, pp.117-136.

De Vroey, M. (1999b), "The marshallian market and the walrasian economy. two incompatible bedfellows", Scottish Journal of Political Economy, vol.46, n³, pp.319-338.

Dos Santos Ferreira, R. (1999), « Keynes et le développement de la théorie de l'emploi dans une économie monétaire ", Working Paper $\mathrm{n}^{\circ} 9905$, BETA, pp.1-56.

Dostaler, G. (1991), "The debate between Hayek and Keynes", in W.J. Barber (éd.), Perspectives on the History of Economic Thought, Aldershot, Edward Elgar, vol.6, pp.77-101.

Dostaler, G. (1999), « Hayek, Keynes et l'économie orthodoxe », Revue d'Économie Politique, vol.109, $\mathrm{n}^{\circ} 6$, pp.762-773.

Foss, N. (1994), "The intertemporal coordination of economic activities : the early debate between Hayek and Keynes", in N. Foss, The austrian 
school and modern economics, Copenhagen, Handelshojskolensforlag, pp.13-39.

Hayek, F. (1928), "Intertemporal price equilibrium and movements in the value of money", in R. McCloughry (éd.), Money, capital and fluctuations. early essays of F.A. Hayek, London, Routledge and Kegan Paul, 1984, pp. 71-117.

Hayek, F. (1931a), "A rejoinder to Mr. Keynes", Economica, vol.11, November, pp.398-403.

Hayek, F. (1931b), «Correspondance avec J.M. Keynes », in The collected writings of John Maynard Keynes, London, Macmillan, 1973, vol.XIII, pp.257-259.

Hayek, F. (1931c), Prices and production, London, Routledge and Sons, édition 1935. Traduction française, Paris, Calmann-Levy, 1975.

Hayek, F. (1931d), "Reflections on the pure theory of money of Mr. J.M. Keynes", Economica, vol.11, August, pp.270-295.

Hayek, F. (1932a), «Correspondance avec J.M. Keynes », in The collected writings of John Maynard Keynes, London, Macmillan, 1973, vol.XIII, pp.260-264.

Hayek, F. (1932b), "Money and capital : a reply", The Econornic Journal, June, vol.42, $\mathrm{n}^{\circ} 166$, pp.237-249.

Hayek, F. (1932c), "Reflections on the pure theory of money of Mr. J.M. Keynes (continued)", Economica, vol.12, February, pp.22-44.

Hayek, F. (1933a), Monetary theory and the trade cycle, New-York, Augustus M. Kelley, 1966.

Hayek, F. (1933b), "Price expectations, monetary disturbances and malinvestment", in Profits, interest and investment, Clifton, Augustus M. Kelley Publishers, 1975.

Hayek, F. (1937), "Economics and knowledge", in F. Hayek, Individualism and economic order, Chicago, The University of Chicago Press, 1948, pp.33-56.

Hayek, F. (1939), Profits, interest and investment, Clifton, Augustus M. Kelley Publishers, 1975.

Hayek, F. (1945), "The use of knowledge in society", The American Economic Review, vol.35, $\mathrm{n}^{\circ} 4$, pp.519-30. Trad. française, Revue Française d'Économie, 1986, vol.5, n², pp.117-140.

Hayek, F. (1966), "Personal recollections of Keynes and the 'keynesian revolution"', The Oriental Economist, vol.34, n663, pp.78-80; in B. Caldwell (éd.), The collected works of F.A. Hayek. contra Keynes and cambridge, London, Routledge, 1995, vol.9, pp.240-246.

Hayek, F. (1978), "Competition as a discovery procedure", in New studies in philosophy, politics, economics, and the history of ideas, London and Henley, Routledge and Kegan Paul, pp.179-190. 
Hayek, F. (1983), "The Keynes centenary : the austrian critique", The Economist, June 11, pp. 45-48; in B. Caldwell (éd.), The collected works of F.A. Hayek. contra Keynes and cambridge, London, Routledge, 1995, vol. 9, pp. 247-255.

Hayek, F. (1988), The fatal conceit. the errors of socialism, London and New York, Routledge. Traduction française, La présomption fatale. les erreurs du socialisme, 1993, Libre Échange, Paris, PUF.

Hicks, J. (1967), "The Hayek story", in Critical essays in monetary theory, Oxford, Clarebdon Press, pp.203-215.

Hicks, J. (1982), "Introductory : LSE and the Robbins circle", in Collected essays in monetary theory. vol. 2, money, interest and wages, Oxford, Basil Blackwell, pp.3-10.

Keynes, J.M. (1930), A treatise on money, 2 vols., in The collected writings of John Maynard Keynes, London, Macmillan, 1973, vol.V-VI.

Keynes, J.M. (1931a), « Correspondance avec F. Hayek », in The collected writings of John Maynard Keynes, London, Macmillan, 1973, vol.XIII, pp.257-260.

Keynes, J.M. (1931b), "The pure theory of money. a reply to Dr. Hayek", Economica, vol.11, November, pp.385-397.

Keynes J.M. (1932a), "Correspondance avec F. Hayek », in The collected writings of John Maynard Keynes, London, Macmillan, 1973, vol.XIII, pp.262-266.

Keynes, J.M. (1932b), «Lettre à J. Robinson », in The collected writings of John Maynard Keynes, London, Macmillan, 1973, vol. XIII, pp. 269-270.

Keynes, J.M. (1934), "Poverty in plenty $:$ is the economic system self-adjusting", The Listener, in The collected writings of John Maynard Keynes, London, Macmillan, 1973, vol.XIII, pp.485-492.

Keynes, J.M. (1936), Théorie générale de l'emploi, de l'intérêt et de la monnaie, édition 1969, Paris, Bibliothèque Scientifique Payot.

Keynes, J.M. (1937), "The general theory of employment", The Quarterly Journal of Economics, vol.51, pp.209-223. Traduction française, Revue Française d'Économie, 1990, vol.5, n% , pp.141-156.

Keynes, J.M. (1973), The collected writings of John Maynard Keynes, D. Moggridge (éd.), London, Macmillan.

Kresge, S. (éd.) (1999), Good money: the new World, The collected works of F.A. Hayek, vol.5, London, Routledge.

Lachmann, L.M. (1986), "Austrian economics under fire : the Hayek-Sraffa duel in retrospect", in W. Grassl et B. Smith (éds.), Austrian economics. historical and philosophical background, New-York, New-York University Press, pp.225-242.

Lawlor, M.S. et B.L. Horn (1992), "Notes on the Sraffa-Hayek exchange", Review of Political Economy, vol.4, n³, pp.314-340. 
Leijonhufvud, A. (1981), Information and coordination, New-York, Oxford University Press.

Leijonhufvud, A. (1998), "Comment : involuntary unemployment one more time", in R. Backhouse et al. (éds.), Economics and methodology. crossing boundaries, London, Macmillan Press, pp.225-236.

McCloughry, R. (éd.) (1984), Money, capital and fluctuations. early essays of F.A. Hayek, London, Routledge and Kegan Paul.

Mongiovi, G. (1990): "Keynes, Hayek and Sraffa : on the origins of chapter 17 of the general theory", Économie Appliquée, vol.XLIII, n², pp.131156.

O'Driscoll, G.P. (1977), "Spontaneous order and the coordination of economic activities", Journal of Libertarian Studies, vol.1, n², pp.137-151.

Pasinetti, L. (1997), "The principle of effective demand", in G. Harcourt et P. Riach (éds.), A 'second edition' of the general theory, London, Routledge, vol.1, pp.93-107.

Robinson, J. (1933), "A parable on savings and investment", Economica, vol.13, February, pp.75-84.

Shackle, G.L.S. (1967), The years of high theory : invention and tradition in economic thought 1926-1939, Cambridge, Cambridge University Press.

Sraffa, P. (1932), "Dr. Hayek on money and capital", Economic Journal, vol.42, $\mathrm{n}^{\circ} 165, \mathrm{pp} .42-53$. Traduction Française par J. Leonard et P. Maurisson, Cahiers d'Économie Politique, 1984, n9, pp.5-17.

Steele, G.R. (1993), "Philosophical perceptions and the precepts of political economy. Friedrich Hayek and John Maynard Keynes", Scottish Journal of Political Economy, vol.40, n²1, pp.89-103.

Tieben, B. (1994), "Misunderstandings and other coordination failures in the Hayek-Keynes controversy", in W. Keizer et al. (éds.), Austrian economics in debate, London, Routledge.

Tobin, J. (1997), "An overview of the general theory", in G. Harcourt et P. Riach (éds.), $A$ 'second edition' of the general theory, London, Routledge, vol.2, pp.3-28. 